\title{
EL VACÍO COMO ELEMENTO ORDENADOR DEL ESPACIO CONSTRUIDO EN LAS UNIVERSIDADES LABORALES: LA PLAZA O PATIO CENTRAL Y los OtRos patios
}

\author{
Miguel Ángel Robles Cardona
}

Empty space as a structuring element of the built spaces in Labour Universities: the main square or courtyard and other courtyards

Boletín Académico. Revista de investigación y arquitectura contemporánea

Escuela Técnica Superior de Arquitectura. Universidade da Coruña

elSSN 2173-6723

unw.boletinacademico.com

Número 4 (2014)

Páginas 23-32

Fecha de recepción 25.10.2013

Fecha de aceptación 21.01.2014

https://doi.org/10.17979/bac.2014.4.0.1006

\section{Resumen}

Las Universidades Laborales nacen con el objetivo de formar a las nuevas generaciones de trabajadores para el desarrollo industrial que se iniciaba en España en los años cincuenta. Llegarían a ser veintiún centros repartidos por todo el país, promovidos por el Estado y diseñados por arquitectos elegidos entre las figuras de máximo prestigio nacional del momento, abarcando su construcción desde 1945 hasta 1976. En el conjunto de proyectos, el vacío destaca por su valor estructurante, bien a modo de plaza o patio de bordes definidos, de explanada o patio abierto o semiabierto, o de intersticios que relacionan unos edificios con otros.

\begin{abstract}
Labour Universities were born with the aim of educating and preparing new generations of workers for the industrial development that began in Spain in the fifties. They would become a group of twenty-one Centers located all over the country, promoted by the Government and designed by architects chosen among the most prestigious national figures of the time, spreading their construction from 1945 through 1976. In all the projects, the empty space stands out for its structuring value either as a square or courtyard of defined edges, or as an esplanade or as an open or half-open courtyard, or as interstices that relate some buildings to each other.
\end{abstract}

\section{Palabras clave}

Universidades Laborales, arquitectura docente, Movimiento Moderno, patio, plaza

\section{Keywords}

Labour Universities, Teaching Architecture, Modern Movement, Courtyard, Square 

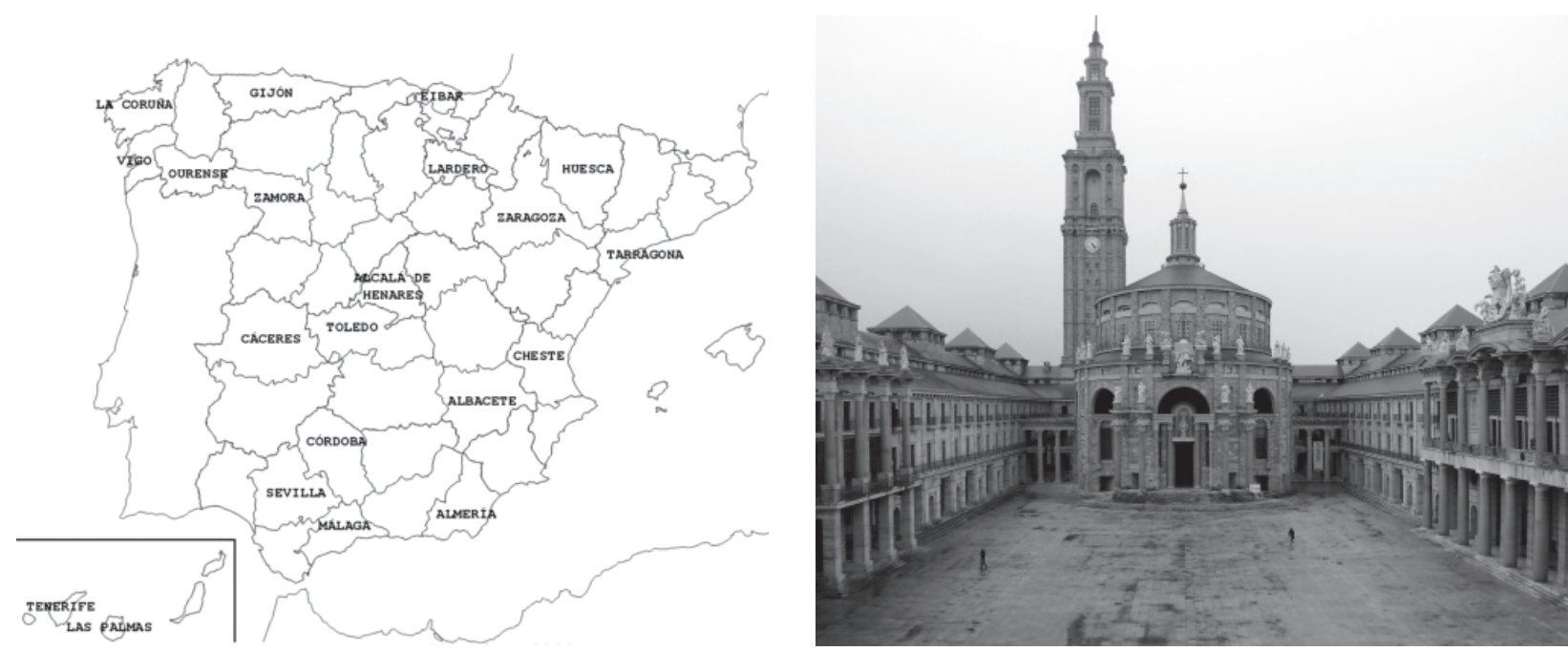

01 Mapa de situación de las veintiuna Universidades Laborales españolas.

02 Luis Moya Blanco (director del proyecto), Pedro Rodríguez y Alonso de la Puente, Ramiro Moya Blanco y Enrique Huidobro Pardo, Universidad Laboral "José Antonio Girón”, Gijón (Asturias), 1946/57; patio central presidido por la capilla.

\section{INTRODUCCIÓN}

Las Universidades Laborales nacieron con el objetivo de formar a las nuevas generaciones en el desarrollo industrial que se iniciaba en España en los años cincuenta, con medios humanos y docentes muy superiores a los de los establecimientos similares de la época. Estaban formadas por un conjunto de instalaciones capaces de dar cabida a miles de alumnos, en régimen de internado en su gran mayoría, procedentes de todo el país y pertenecientes, en general, a las clases sociales más desfavorecidas.

Su actividad docente queda enmarcada entre 1955 y 1978, si bien el primer proyecto —el de la Universidad Laboral de Gijón - es encargado a finales de 1945; y la última en entrar en funcionamiento, en octubre de 1976, es la de Vigo.

Su artífice y creador intelectual fue el Ministro de Trabajo José Antonio Girón de Velasco, abandonándose a partir de su salida del Gobierno en 1957 los planteamientos megalómanos que caracterizaron a los primeros centros. Los nuevos se proyectaron bajo un férreo control económico que daría lugar a conjuntos más pequeños, compactos y austeros.

Llegarían a ser un total de veintiuna Universidades Laborales $^{1}$ repartidas por todo el territorio nacional (Fig. 01), promovidas por el Estado y diseñadas por diferentes arquitectos o agrupaciones de estos, elegidos entre las figuras de máximo prestigio del panorama arquitectónico del momento, como Luis Moya
Blanco, Fernando Moreno Barberá, Luis Laorga Gutiérrez, José López Zanón o Julio Cano Lasso.

Dado el gran número de proyectos realizados y el amplio elenco de profesionales que intervinieron en los mismos, podría abordarse el análisis de estos conjuntos desde múltiples puntos de vista. Este artículo se propone explicar el valor que el espacio libre -el vacío, lo no construido - adquiere como elemento ordenador, incluso a veces estructurante, de los programas de las Universidades Laborales, a partir de las distintas maneras en que éste se formaliza en los diferentes conjuntos.

\section{GRANDES VACÍOS CENTRALES QUE ESTRUCTURAN CONJUNTOS DE BORDES INDETERMINADOS}

En una primera categoría es posible encuadrar aquellos centros en los que aparece un gran vacío principal - sea éste un patio o una plaza-, construido, delimitado, definido y cerrado, que es el elemento fundamental que ordena el conjunto, centro neurálgico, lugar de encuentro y paso y, en definitiva, síntesis que explica la estrategia de proyecto.

De este modo, en la Universidad Laboral de Gijón la plaza central queda perimetrada por las partes arquitectónicamente nobles, y rodeada a su vez por otras más funcionales, sirviendo para la expansión de los alumnos y como espacio de celebraciones ${ }^{2}$. Es al acceder a ella, a través del atrio corintio situado en su esquina sureste, cuándo se descubre la verdadera fachada del edificio, que es interna (Fig. 02). 

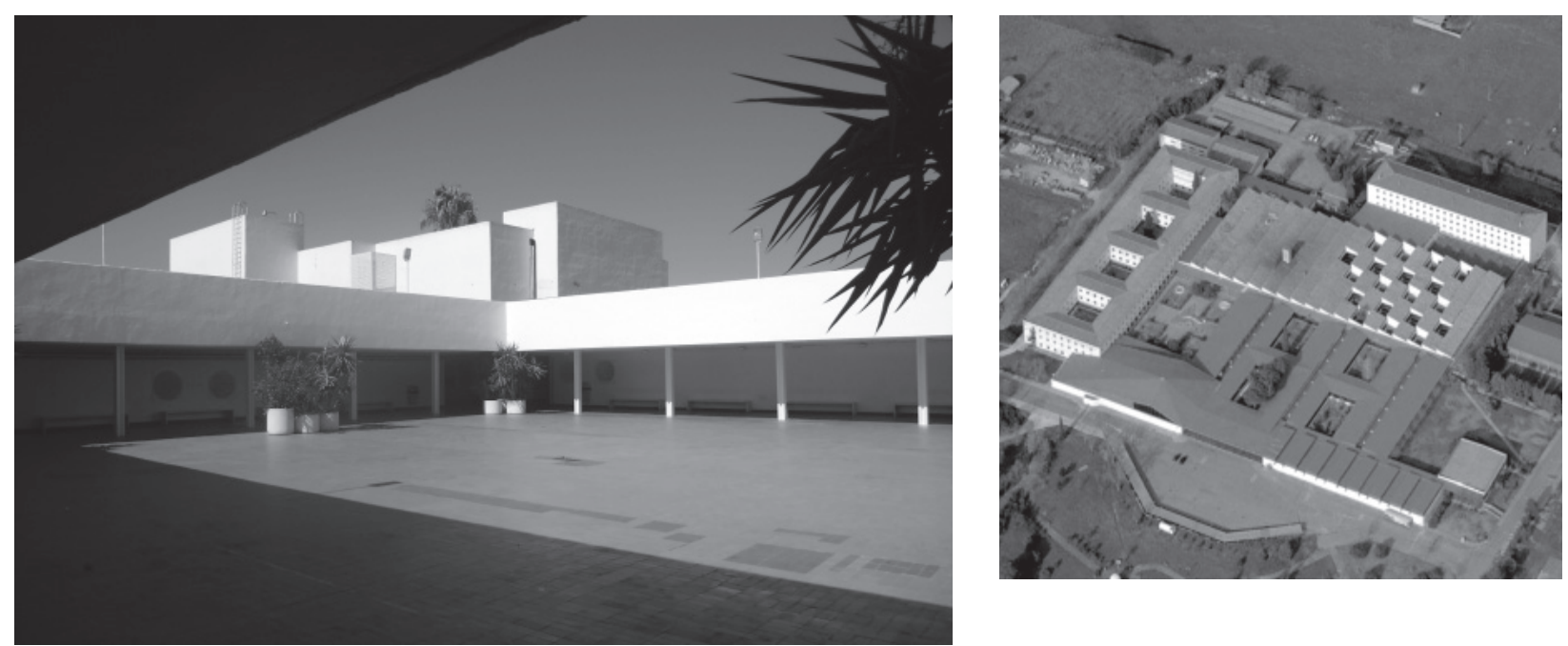

03 Julio Cano Lasso (director del proyecto), Alberto Campo Baeza, Antonio Más-Guindal Lafarga y Miguel Martín Escanciano, Centro de Universidades Laborales, Almería, 1973/74; patio central.

04 Luis Laorga Gutiérrez y José López Zanón, Universidad Laboral «Hispano-Americana», Cáceres, 1964/67; vista aérea.

Este amplio espacio vacío, cerrado por pabellones a modo de plaza mayor, que es a la vez patio, se configura como la pieza principal del conjunto. Se podría decir que, en cierto modo, el edificio se compone de la plaza central y su alrededor. Ésta muestra el orden, la composición, y desde su precisa y nítida imagen el conjunto se extiende como una masa de borde aleatorio, producto de la agregación de nuevas fábricas cerrando patios. Es decir, la Universidad Laboral es una trama de vacíos que da lugar a una forma indeterminada, amorfa, porque está obtenida por la agregación de elementos que se han ido incorporando hasta completar el programa, pero que podría ser indefinida. Estos patios cuadrangulares, de diverso tamaño y proporción, destinados cada uno a un fin, establecen el orden y la separación de las distintas funciones, enlazando el edificio con la naturaleza, iluminando y ventilando las estancias.

Un mecanismo similar al empleado en Gijón, dentro de un lenguaje arquitectónico de absoluta modernidad, se produciría casi dos décadas más tarde en el Centro de Universidades Laborales de Almería, compuesto como un organismo complejo, como una red de calles y plazas articuladas ${ }^{3}$ (Fig. 03). Su estructura funcional surge a partir de la intersección de dos pares de ejes que se cruzan perpendicularmente formando en su encuentro una gran plaza, espacio exterior controlado, cerrado y delimitado. En ella confluyen todas las circulaciones, configurándose como un lugar natural de encuentro y relación de más de $1000 \mathrm{~m} 2$ de superficie, útil además para reuniones y actos al aire libre, centro de distribución y de vida. "Podríamos decir que, casi más que unos ejes, hay un primer espacio que organiza, que es el espacio público»" ${ }^{4}$. Es por tanto, desde el vacío de esta gran plaza, desde dónde se puede comprender el funcionamiento del edificio.

El doble par de ejes que se cruzan formando la plaza central se prolonga en las cuatro direcciones ortogonales conectando una red de patios secundarios, de espacios públicos introvertidos que "como nudos en una malla, agrupan en torno a sí los locales ${ }^{5}$. Estos nudos generan una estructura de orden superior en la que vacíos y llenos se complementan, convirtiendo estos patios cerrados, de dimensiones variables según su uso y función, en «habitaciones abiertas al cielo» ${ }^{6}$.

Destaca la trama en damero de aulas vinculadas a pequeños patios privados que a su vez se cierran a los corredores de distribución. "Se rechaza esta transparencia en busca de una sofisticada complejidad espacial, como ocurre con el urbanismo árabe: en el que lo público se configura con tapias blancas, y la arquitectura se abre al interior de los patios» ${ }^{7}$ procurando, de otra parte, un mejor aislamiento acústico y visual entre las zonas de descanso y de docencia. «En definitiva el proyecto se basa en una esencial forma de tratar el espacio arquitectónico, incorporando a la arquitectura los espacios no edificados, que se organizan en forma de patios y plazas» ${ }^{8}$. El conjunto se proyecta, por tanto, desde el vacío, desde los lugares de relación, siendo el núcleo fundamental la gran plaza central. 

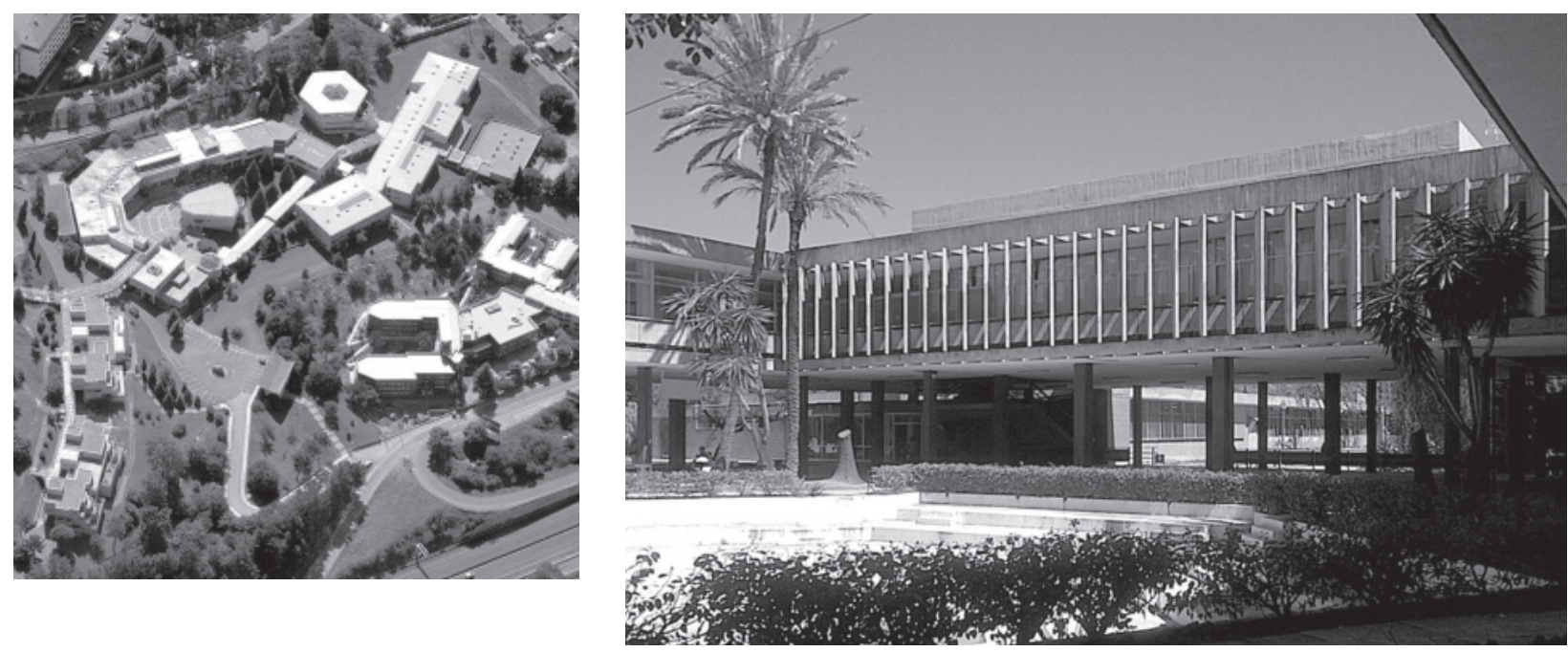

05 Luis Laorga Gutiérrez y José López Zanón, Universidad Laboral «Quinto Sertorio», Huesca, 1964/67; vista aérea. 06 Fernando Moreno Barberá, Centro de Universidades Laborales «Utrera Molina», Málaga, 1972/78; patio del edificio de dirección.

\section{CONJUNTOS CERRADOS DE BORDES DEFINIDOS ORDENADOS EN TORNO A UN GRAN PATIO}

Otros proyectos se estructuran también a partir de un gran vacío, si bien ahora estos patios o plazas principales forman parte de conjuntos con un perímetro definido, prefijado, no siendo posible como en los casos anteriores, por tanto, modificar su crecimiento sin variar su esencia.

Estos proyectos muestran una disposición cerrada, casi ciega al exterior y abierta a patios interiores de diferente tamaño y función, respondiendo a la necesidad de defenderse de las inclemencias del tiempo y como manera de disminuir los recorridos peatonales y los trazados de las instalaciones, mejorando a su vez el control sobre el alumnado. Por otra parte, su trazado responde a una actitud conservadora y academicista, fiel a la tradición española de composición, cerrando patios dentro de una forma general definida, como ocurre en El Escorial.

De este modo, en la Universidad Laboral de Cáceres, el patio principal ordena y relaciona las partes de mayor jerarquía del programa, acusando un carácter más público por su posición junto al vestíbulo de acceso al edificio9 (Fig. 04). Colindando con dicho patio, en el ala este del conjunto, se sitúa el internado, organizado en cuatro colegios de planta cuadrada en torno a sendos patios. Por su parte, al otro lado del patio principal se ubica el conjunto de aulas y laboratorios, ordenado alrededor de cuatro patios de la misma dimen- sión. Posteriormente, en la transformación sufrida por los talleres para convertirlos en aulas, éstos quedarían vinculados a pequeños patios privativos en una estructura de damero similar a la del centro almeriense, si bien aquí no es posible acceder a ellos desde las aulas, quedando por el contrario abiertos a los corredores.

De otro lado, en la Universidad Laboral de Huesca el Patio Principal queda remetido en la planta respecto al acceso general, de manera que se vincula fundamentalmente a las piezas docentes abiertas a él ${ }^{10}$ (Fig. 05). También aquí las aulas se abren a pequeños patios con vegetación que las aíslan del ruido exterior, aunque vinculadas nuevamente a los pasillos generales.

\section{LA PLAZA PORTICADA COMO NÚCLEO COMPACTO DE UN CONJUNTO DISGREGADO}

Con el Centro de Universidades Laborales de Málaga aparece una nueva variación de proyecto ordenado a partir de un espacio vacío, componiendo el conjunto como un gran disgregado de edificios ${ }^{11}$ (Fig. 06). En este caso es el cuerpo de dirección - pabellón principal que adopta la tipología claustral — el que actúa como aglutinante y síntesis de la estrategia de implantación utilizada por el proyectista. Su planta baja diáfana define una especie de plaza porticada y un porche perimetral para esparcimiento, descanso y paseo de los alumnos, así como para la organización de los accesos a los diferentes pabellones docentes que con él se articulan. En el área central de este volumen, el edificio se descubre al cielo construyendo un graderío para la 

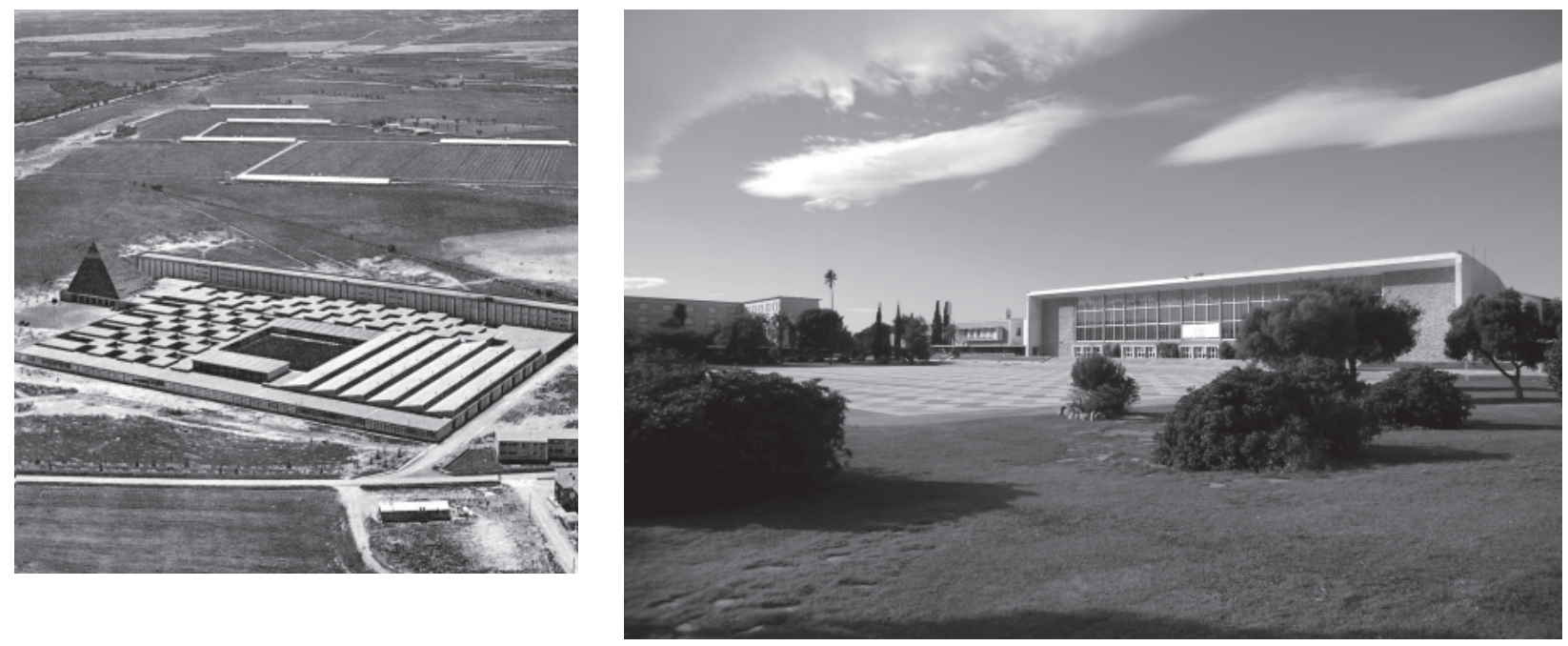

07 José Antonio López Candeira, Centro de Universidades Laborales de Vigo, Pontevedra, 1975/76; vista aérea.

08 Manuel Sierra Nava, Luis Peral Buesa y Antonio de la Vega Martínez, Universidad Laboral «Francisco Franco», Tarragona, 1950/58; explanada central presidida por el edificio del comedor. Los seis bloques de planta en L que acogen los colegios-residencia se disponen, tres a tres, a cada lado de esta plaza.

celebración de actos al aire libre, deprimiendo su cota respecto a la del porche perimetral para permitir visuales ininterrumpidas desde cualquier punto del deambulatorio cubierto.

Por otra parte, en el Centro de Universidades Laborales de Vigo aparece también una plaza porticada, y en torno a ella, más dispersos cuanto más alejados, se sitúan el resto de edificios del conjunto ${ }^{12}$ (Fig. 07). Se produce, en cierto modo, el mismo mecanismo proyectual que en Málaga: esto es, un espacio central público - expresión de la estrategia de proyecto-y un conjunto de piezas aisladas y adaptadas a la topografía según un criterio paisajista que no atiende, en esta ocasión, a malla ortogonal alguna. Aquí el vacío es perfilado, de un lado, por un edificio longitudinal quebrado, contenedor continuo de las funciones lúdico-asistenciales, que se pliega sobre sí mismo cerrando la superficie cóncava del atrio. En el punto focal de la plaza se sitúa la sala polivalente, que ocupa el lugar de la tradicional iglesia en los pueblos. Por otra parte, el límite inferior del recinto quedaría definido por una galería cubierta de circulación.

\section{LA EXPLANADA QUE QUERÍA SER PLAZA, Y A VECES LO ERA}

Existen conjuntos en los que el vacío principal que ordena el proyecto se configura, no a modo de patio como lugar construido y cerrado por edificación, sino como una explanada que, si bien adquiere la personalidad urbana de una plaza, se caracteriza por su carácter abierto y sus bordes discontinuos.
Según este planteamiento se organiza la Universidad Laboral de Tarragona, en la que la avenida principal de acceso al conjunto enlaza tangencialmente con la gran plaza que lo ordena, presidida por la nave del comedor $^{13}$ (Fig. 08). A su vez, los seis edificios de residencia, de cuatro alturas y planta en forma de L, se disponen tres a tres a cada lado de la explanada, cerrando a su vez pequeños espacios ajardinados. Por último, alineado a eje con el edificio del comedor, al otro lado de la plaza, se sitúa el grupo escolar. De este modo, la explanada se convierte en un tapiz pensado para que en cada vértice un chico pueda hacer gimnasia, en número suficiente para todos los alumnos de la Universidad Laboral ${ }^{14}$.

De un modo similar, en la Universidad Laboral de Córdoba pueden distinguirse dos grandes vacíos ${ }^{15}$ (Fig. 09). En primer término, al fondo del eje de acceso, la plaza representativa o de llegada cerrada por el paraninfo y la iglesia. A continuación, la gran explanada central, escenario de todos los actos solemnes. A cada lado de esta plataforma perfilada por pérgolas y galerías se disponen, simétricamente respecto a un eje axial, los seis colegios-residencia con sus plantas cruciformes. Al fondo de la plaza, cerrando la perspectiva, se sitúa el edificio de usos comunes, que contiene los comedores y demás locales de servicio. Por último, en el extremo opuesto de este eje procesional, apartado de la rigidez geométrica general, se ubican la iglesia y su torre.

En la Universidad Laboral de Sevilla volvería a repetirse el mecanismo, como en Córdoba, de un gran eje de 

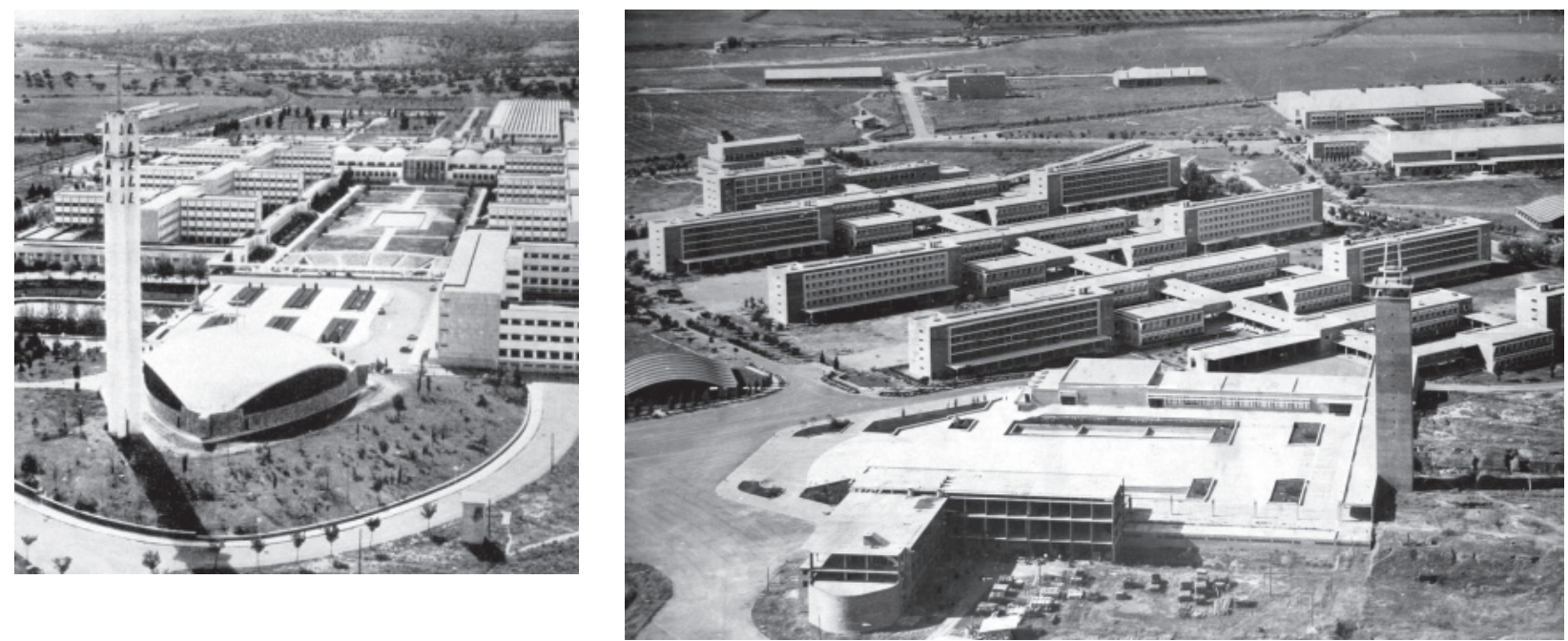

09 Francisco Robles Giménez, Daniel Sánchez Puch, Manuel de los Santos Nicolás y Fernando Cavestany y Pardo-Valcárcel, Universidad Laboral «Onésimo Redondo», Córdoba, 1952/56; vista aérea.

10 Grupo OTAISA (Oficinas Técnicas de Arquitectura e Ingeniería, S.A.), integrado por Luis Gómez Estern, Alfonso Toro Buiza, Rodrigo Medina Benjumea y Felipe Medina Benjumea, Universidad Laboral "José Antonio Primo de Rivera», Sevilla, 1952/65; vista aérea.

llegada que desemboca en una explanada de acceso, en torno a la que se disponen las piezas representativas del conjunto, de modo que éstas configuran una $U$ atada por una galería cubierta y continua que define su borde $^{16}$ (Fig. 10). Desde ésta arranca la columna central de distribución, que se curva enlazando los edificios docentes y residenciales a modo de espina de pez, y a la que se conectan, a su vez, los patios de recreo, plazas y jardines para la expansión de los alumnos vinculados a cada colegio-residencia. Asimismo, a continuación de la plaza de acceso se sitúa el vacío poligonal que acoge la parada de autobuses.

Por su parte, la Universidad Laboral de A Coruña se estructura de manera similar a la de Sevilla: una amplia explanada o plaza de acceso y una trama de espacios llenos y vacíos que se extiende, esta vez hacia ambos lados de aquella ${ }^{17}$ (Fig. 11). El conjunto se organiza, por tanto, como una retícula de calles y plazas con pabellones aislados volumétricamente, pero conectados a través de porches cubiertos y de espacios al aire libre, de manera que éstos adquieren gran autonomía formal y funcional. En el centro del conjunto se sitúa la gran plaza de $3500 \mathrm{~m} 2$ de superficie que, mirando a la ría (Fig. 12), reúne en torno a sí los usos colectivos de la capilla, los comedores y el salón de actos con su gran vestíbulo. Desde esta plaza central, lugar de reunión y acceso al centro, arrancan las calles y circulaciones que distribuyen la retícula hipodámica que define las pautas para su posible crecimiento. El tamańo de esta plaza remarca la dimensión del edificio, contribuyendo a su compresión como un todo.

\section{UNA SOLUCIÓN INTERMEDIA}

En la Universidad Laboral de Zamora se produce una solución particular a caballo entre aquellas que cuentan con una plaza de acceso - esta vez de una escala mucho más contenida- y las que albergan un gran patio interior dentro de un conjunto de organización más o menos compacta y edificación continua $^{18}$ (Fig. 13). Este proyecto se dispone -que no se organiza- en torno a un gran jardín a modo de espacio privado al aire libre, al que se accede desde el zaguán en su esquina. Aquí el vacío no constituye realmente un patio en su concepto tradicional, es decir, un elemento claustral. No es, por tanto, una plaza, ni está definido por el diálogo entre elementos principales que lo toman como soporte, como ocurre en Gijón. Se trata de un gran espacio interior de juegos y jardines cerrado por los pabellones que componen el conjunto, pero no es preciso circular por él, ni atravesarlo, para utilizar el edificio. Es por tanto, un vacío entre las partes que lo delimitan, que no genera las leyes compositivas del proyecto. De hecho es un patio abierto desde el punto de vista proyectual, que se cierra en su cuarto lado con un pabellón trasero —un simple frontón cubierto-, de modo que su espacio queda definido por completo. Así, en el conjunto de Zamora lo básico es lo lleno; y el vacío - pese a su función ordenadora—, se obtiene como resultado ${ }^{19}$.

Por otra parte, el edificio abre una plaza de acceso en esquina que sale a ordenar su presencia en la ciudad, sirviendo de conexión con ésta. Se trata de un gesto 


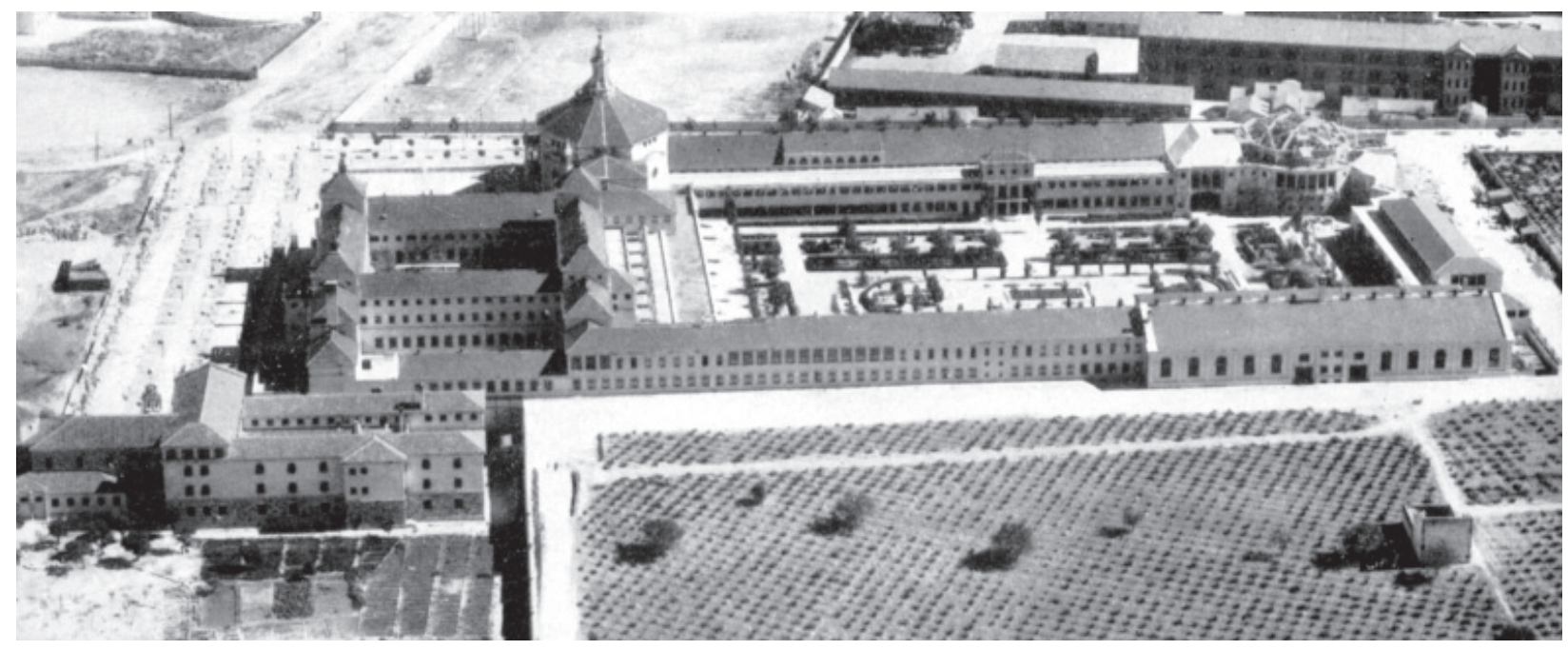

13 Luis Moya Blanco (director del proyecto), Pedro Rodríguez y Alonso de la Puente, Ramiro Moya Blanco y Enrique Huidobro Pardo, Universidad Laboral «San José», Zamora, 1947/57; vista aérea.

12 Luis Laorga Gutiérrez y José López Zanón, Universidad Laboral «Crucero de Baleares», A Coruña, 1960/67; vista aérea

11 Universidad Laboral de A Coruña; plaza central o de llegada.
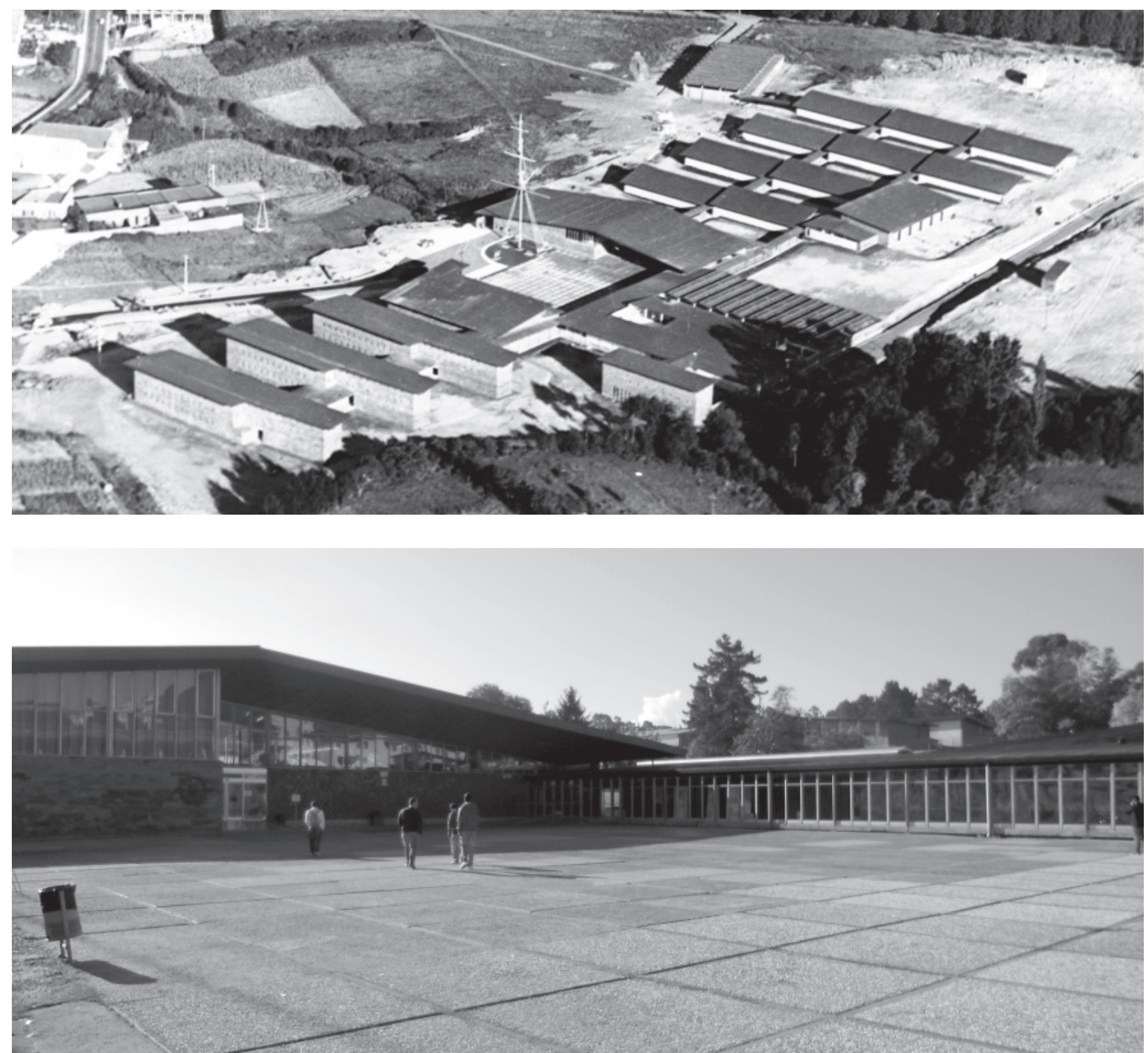

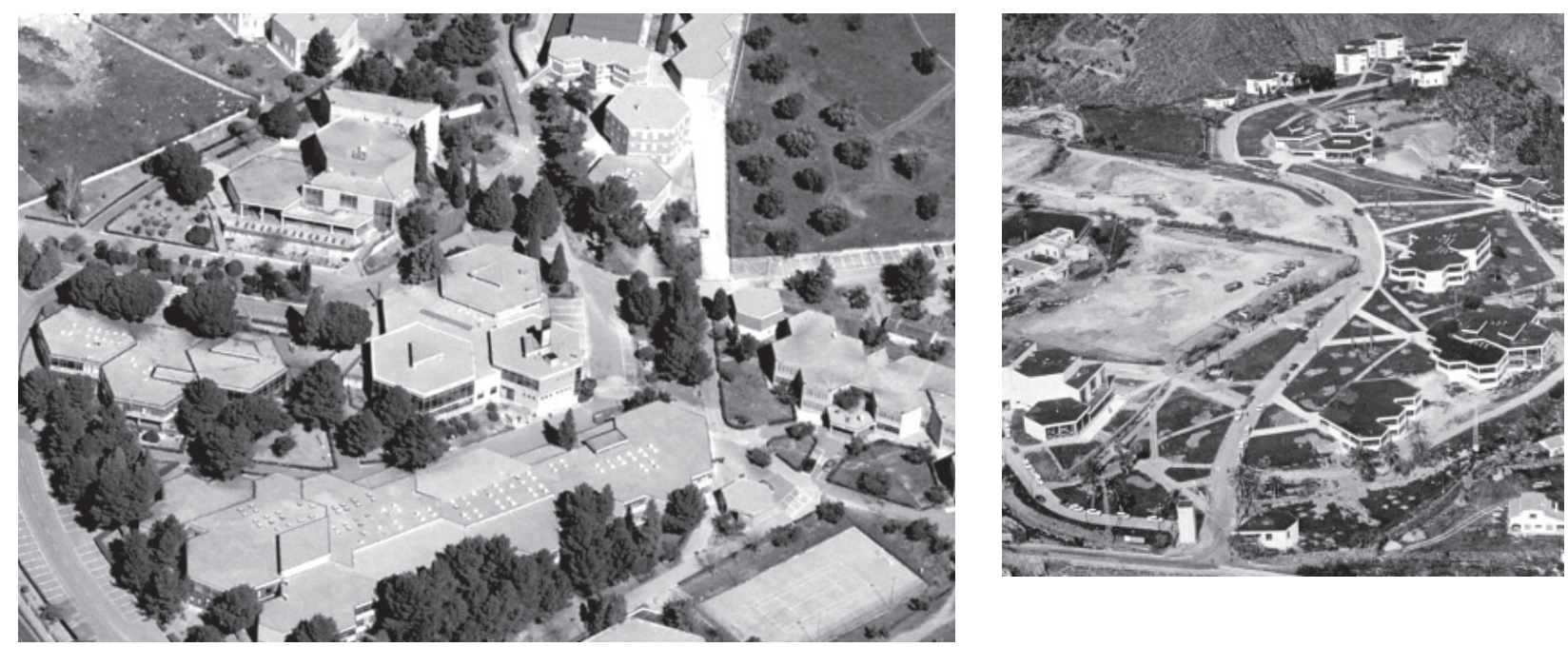

14 Fernando Moreno Barberá, Centro de Universidades Laborales «Blas Tello», Toledo, 1971/77; vista aérea.

15 Fernando Moreno Barberá, Centro de Universidades Laborales «Licinio de la Fuente», Las Palmas de Gran Canaria, 1971/73; vista aérea.

extraído de la tradición para conceder el necesario relieve urbano al templo y a su entrada cuando éste se sitúa, como aquí, en una retícula de ciudad sin plazas ni espacios libres principales. Por último, el pabellón destinado a residencia de internos forma una $\mathrm{C}$ en torno a un patio cuadrado, a modo de claustro, con un jardín de carácter íntimo cerrado en su cuarto lado por el pabellón de la comunidad de religiosos que regía el centro.

\section{LA DISOLUCIÓN DEL ESPACIO LIBRE O EL VACÍO DERRAMADO}

Es posible, por último, agrupar otros proyectos en los que, si bien pueden distinguirse espacios con vocación de plaza urbana, éstos se configuran con un carácter abierto que los convierte, ciertamente, en ámbitos más o menos delimitados por la agrupación en proximidad de un conjunto de pabellones. Se trata de los Centros de Universidades Laborales de Toledo y de Las Palmas ${ }^{20}$ (Fig. 14-15). En ellos, la jerarquía de los diferentes espacios públicos creados viene definida por las características de un urbanismo medieval; es decir, no existen ejes ni perspectivas orientadas hacia elementos aislados, sino que al recorrer los conjuntos se recibe la impresión de sucesivas calles estrechas y plazas amplias, produciendo ritmos longitudinales y transversales. En definitiva, se trata de composiciones de arquitecturas colocadas sobre el terreno con un cierto criterio paisajista, desinhibido en su componente social respecto a la rigidez que acusaban los primeros conjuntos.
En estos proyectos, siguiendo el camino de entrada se llega a un espacio público de límites difusos rodeado por los edificios de dirección, de comedores y cocinas, y del teatro y cafetería, creando el ambiente urbano de una plaza a la que todos se abren y desde la que a todos se accede. Es decir, se disponen próximas a la entrada las zonas en las que los alumnos podrían encontrarse con los visitantes de manera que éstos no pudiesen perturbar el funcionamiento del centro, creando, por tanto, en el conjunto, diferentes niveles de intimidad. Además, la posición central de estas piezas, entre la zona residencial y la docente, hace que estos edificios de uso común sean fácilmente accesibles desde ambas. Por último, la unidad residencial se dispone en el área más elevada del solar configurando una especie de plaza alrededor de la capilla.

\section{A MODO DE COLOFÓN}

Se ha comprobado, por tanto, cómo en las Universidades Laborales españolas el espacio vacío - lo no construido, bien se manifieste a modo de plaza o patio de bordes perfectos y definidos, bien como una explanada o patio de carácter abierto o semiabierto, bien como una serie de intersticios que ponen en relación unos edificios con otros- es el que asume el valor de elemento que estructura el programa y las relaciones que se producen entre sus distintas partes. Y esto se consigue agrupando siempre en torno a sí — sea como fuere su formalización concreta- las piezas construidas de mayor jerarquía, es decir, las que acogen las dependencias de dirección o los usos comunes del teatro o aula magna, los comedores y, en algunos casos, la capilla. 


\section{Notas}

1. Las veintiuna Universidades Laborales proyectadas y construidas, por orden cronológico, así como sus autores, son: Universidad Laboral "José Antonio Girón” de Gijón (1946/57), arquitectos: Luis Moya Blanco como arquitecto director, y su equipo formado por Pedro Rodríguez y Alonso de la Puente, Ramiro Moya Blanco y Enrique Huidobro Pardo; del mismo equipo es la Universidad Laboral "San José" de Zamora (1947/57); Universidad Laboral "Francisco Franco" de Tarragona (1950/58), arquitectos: Manuel Sierra Nava, Luis Peral Buesa y Antonio de la Vega Martínez; Universidad Laboral "Onésimo Redondo" de Córdoba (1952/56), arquitectos: Francisco Robles Giménez, Daniel Sánchez Puch, Manuel de los Santos Nicolás y Fernando Cavestany y Pardo-Valcárcel; Universidad Laboral "José Antonio Primo de Rivera" de Sevilla (1952/65), arquitectos: grupo OTAISA (Oficinas Técnicas de Arquitectura e Ingeniería, S. A.), integrado por Luis Gómez Estern, Alfonso Toro Buiza, Rodrigo Medina Benjumea y Felipe Medina Benjumea; Universidad Laboral “Crucero de Baleares” de A Coruña (1960/67), arquitectos: Luis Laorga Gutiérrez y José López Zanón; Universidad Laboral de Alcalá de Henares, Madrid (1964/66), arquitecto: Martín José Marcide Odriozola; Universidad Laboral "Hispano-Americana" de Cáceres (1964/67), arquitectos: Zanón y Laorga; del mismo equipo es la Universidad Laboral "Quinto Sertorio" de Huesca (1964/67); Universidad Laboral "Virgen del Pilar" de Zaragoza (1964/70), arquitecto: Manuel Ambrós Escanellas; Centro de Orientación de Universidades Laborales "Jesús Romeo" de Cheste, Valencia (1965/69), arquitecto: Fernando Moreno Barberá; Centro Técnico Laboral de Éibar, Guipúzcoa (1966/73), arquitecto: Álvaro Líbano Pérez-Ulibarri; Colegio de Promoción Social de Universidades Laborales de Tenerife (1969/77), arquitectos: Vicente Saavedra Martínez y Javier Díaz-Llanos La Roche; Centro de Universidades Laborales "Licinio de la Fuente" de Las Palmas de Gran Canaria (1971/73), arquitecto: Fernando Moreno Barberá; del mismo arquitecto es el Centro de Universidades Laborales "Blas Tello" de Toledo (1971/77); Centro de Universidades Laborales de Almería (1973/74), arquitecto: Julio Cano Lasso dirige al equipo formado por Alberto Campo Baeza, Antonio Más-Guindal Lafarga y Miguel Martín Escanciano; Centro de Universidades Laborales "Utrera Molina" de Málaga (1972/78), arquitecto: Fernando Moreno Barberá; Centro de Universidades Laborales de Lardero, Logroño (1973/74), arquitectos: Julio Cano Lasso en colaboración con Ramón Campomanes Grande; de los mismos arquitectos es el Centro de Universidades Laborales de Albacete (1974/75); Centro de Universidades Laborales de Orense (1974/76), arquitectos: Julio Cano Lasso en colaboración con José Manuel Sanz Sanz y Antonio Ortiz Carvajal; Centro de Universidades Laborales de Vigo, Pontevedra (1975/76), arquitecto: José Antonio López Candeira.

2. Para ampliar la información sobre la Universidad Laboral de Gijón puede consultarse: Luis Moya Blanco et al., "Universidad Laboral José Antonio Girón, en Gijón. Sesión crítica de arquitectura”, Revista Nacional de Arquitectura 168 (1955): 35-48; Antón González-Capitel, "La Universidad Laboral de Gijón o el poder de las arquitecturas", Arquitecturas Bis 12 (1976): 25-31; Carlos Montes Serrano, "Clasicismo, licencia y retórica en la arquitectura de Luis Moya: a propósito del 50 aniversario de la primera piedra de la Universidad Laboral de Gijón”, Ra: Revista de Arquitectura 3 (1999): 63-74; Ángel Martín Rodríguez et al., Los Talleres de la Universidad Laboral de Gijón (Gijón: CICEES, 2006); Sergio Ríos González y César de Castro Valdés, La Laboral de Gijón: de orfanato minero a ciudad de la cultura (Asturias: Ménsula, 2008).

3. Para ampliar la información sobre el Centro de Universidades Laborales de Almería puede consultarse: Elisa Valero Ramos, Universidad Laboral de Almería, 1971-1974. J. Cano, A. Campo, d. Martín, A. Más (Almería: Ediciones del Colegio de Arquitectos de Almería, 2008); Julio Cano Lasso y Miguel Centellas Soler, "Universidad Laboral de Almería", Periferia 12 (1993): 14-33.

4. Declaraciones de Diego Cano Pintos, arquitecto e hijo de Julio Cano Lasso, al autor de este artículo en su oficina, situada en la antigua casa-estudio de su padre en la Urbanización La Florida de Madrid, el 8 de marzo de 2012.

5. Valero, Universidad Laboral de Almería, 30.

6. Ibídem, 32.

7. Ibídem, 63.

8. Ibídem, 100 .

9. Para ampliar la información sobre la Universidad Laboral de Cáceres puede consultarse: Carlos Barrantes López, "La Universidad Laboral de Cáceres, un ejemplo significativo de arquitectura escolar extremeña en la década de los sesenta", Ars et Sapientia: Revista de la Asociación de Amigos de la Real Academia de Extremadura de las Letras y las Artes 14 (2004): 25-47.

10. Para ampliar la información sobre la Universidad Laboral de Huesca puede consultarse: Luis Laorga Gutiérrez y José López Zanón, "Universidad Laboral de Huesca - España”, Informes de la Construcción 227 (1971): 33-43.

11. Para ampliar la información sobre el Centro de Universidades Laborales de Málaga puede consultarse: Miguel Ángel Robles Cardona, Proyecto y Topografia: Universidad Laboral de Málaga (Barcelona: del autor, 2011).

12. Para ampliar la información sobre el Centro de Universidades Laborales de Vigo puede consultarse: Andrés Perea Ortega, "Sobre la Universidad Laboral de Vigo. Una obra de José Antonio López Candeira", Obradoiro 4-5 (1979): 46-61; "Universidad Laboral: Vigo (Espańa)", Panorámica de la Construcción, Arquitectura y Diseño 44 (1981): 41-42; Antonio Río Vázquez, Las Universidades Laborales gallegas. Arquitectura y modernidad (Santiago de Compostela: Colexio Oficial de Arquitectos de Galicia, 2011), 116-131.

13. Para ampliar la información sobre la Universidad Laboral de Tarragona puede consultarse: Xavier Monteys Roig et al., La Universidad Laboral de Tarragona 1952-1956 (Tarragona: COAC, 2006); Josep María Buqueras i Bach y Ricardo Zafrilla Tabarra, La Laboral de Tarragona: fonaments i construcción (Tarragona: Colegio de Aparejadores y Arquitectos Técnicos de Tarragona/Silva, 2007).

14. Cf. Entrevista realizada al arquitecto Luis Peral Buesa por el arquitecto Xavier Monteys Roig, recogida en: Monteys, La Universidad Laboral de Tarragona. 15. Para ampliar la información sobre la Universidad Laboral de Córdoba puede consultarse: Fernando Cavestany y Pardo-Valcárcel et al., "Proyecto de Universidad Laboral en Córdoba", Revista Nacional de Arquitectura 138 (1953): 1-10; Idem, "Iglesia en la Universidad Laboral de Córdoba", Arquitectura 52 (1963): 18-19.

16. Para ampliar la información sobre la Universidad Laboral de Sevilla puede consultarse: Guillermo Pavón Torrejón y Fernando Quiles García, "La Universidad Laboral de Sevilla, arquitectura en el paisaje", Atrio 10-11 (2005): 125-32; José Joaquín Parra Bañón, "Pesquisas sobre la Universidad Laboral de Sevilla: apología, vigencia y ruina", Revista de historia y teoría de la arquitectura 8 (2006): 91-131; Idem., "Dos fragmentos de OTAISA en la Universidad Laboral de Sevilla: cinco gimnasios y un taller en agonía", Ra: Revista de Arquitectura 10 (2008): 69-82; Idem., "Arquitectura y autopsia: análisis gráfico de algunos restos de la inexistente Universidad Laboral de Sevilla", EGA. Revista de Expresión Gráfica Arquitectónica 13 (2008): 36-37.

17. Para ampliar la información sobre la Universidad Laboral de A Coruńa puede consultarse: Carlos de Miguel, "Concurso de proyectos para la Universidad Laboral de La Coruña", Arquitectura 31 (1961): 19-36; Xan Casabella López, "A Universidade Laboral da Coruńa no contexto das UU.LL. de España”, Obradoiro 17 (1990): 34-39; Idem., "Universidad Laboral Crucero Baleares" en: Celestino García Braña y Fernando Agrasar Quiroga, Arquitectura Moderna en Asturias, Galicia, Castilla y León. Ortodoxia, márgenes y transgresiones (Oviedo: Colegios Arquitectos Asturias, Galicia, León y Castilla y León Este, 1998), 146-49; Río, Las Universidades Laborales gallegas.

18. Para ampliar la información sobre la Universidad Laboral de Zamora puede consultarse: Luis Moya Blanco et al., «Proyecto de Escuelas Salesianas de San José en Zamora. Memoria (Madrid: 1947)», en: Galo Sánchez Sánchez, La Universidad Laboral de Zamora: Una manifestación del proyecto social y educativo del franquismo: (1946-80) (Zamora: Instituto Estudios Zamoranos Florián Ocampo, Diputación de Zamora, 2006), 245-258; Idem., "Capilla de las Escuelas Profesionales Salesianas de San José, en Zamora", Revista Nacional de Arquitectura 151-152 (1954): 57-64; Idem., "Fundación San José, en Zamora", Revista Nacional de Arquitectura 161 (1955): 1-11; Antón González-Capitel, "La Universidad Laboral de Zamora", BAU: Revista de arquitectura, urbanismo, arte y diseño 2-3 (1990): 130-155.

19. Cf. Antón González-Capitel, La arquitectura de Luis Moya Blanco (Madrid: Colegio Oficial de Arquitectos de Madrid, 1982), 175. 
20. Sobre los Centros de Universidades Laborales de Toledo y de Las Palmas no existe bibliografía específica, si bien aparecen referidos en: Juan Blat Pizarro, Fernando Moreno Barberá. Modernidad y arquitectura (Barcelona: Fundación Caja de arquitectos, 2006), 119-126, 250; Juan Bravo Bravo, Enseñanzas prácticas. Espacios para la docencia y la investigación en la obra de Fernando Moreno Barberá (Directora: Carmen Jordá Such) (Valencia: Departamento de Composición Arquitectónica de la Escuela Técnica Superior de Arquitectura de Valencia-Universidad Politécnica de Valencia, 2007).

\section{Procedencia de las ilustraciones}

Fig. 01-03, 06, 08 y 12. Elaboración propia, 2012/13.

Fig. 04, 07 y 14. Consulta: 1 de abril de 2013. Disponible en: http://www.bing.com/mapas.

Fig. 05. Archivo del I.E.S. Pirámide, antigua Universidad Laboral de Huesca. Archivo sin organizar.

Fig. 09, 13 y 15. José Utrera Molina, Nuevo horizonte de las Universidades Laborales (Madrid: Ministerio de Trabajo, Servicio de Publicaciones, 1970).

Fig. 10. Archivo de la Universidad Pablo de Olavide, antigua Universidad Laboral de Sevilla. Archivo sin organizar.

Fig. 11. Archivo del I.E.S. Universidade Laboral, antigua Universidad Laboral de A Coruńa. Archivo sin organizar.

\section{Sobre el autor}

Tras obtener el título de Arquitecto por la ETSA de Sevilla en 2009, realiza el Máster en Teoría y Práctica del Proyecto de Arquitectura en la ETSA de Barcelona durante el año académico 2010/11. De ahí resulta la publicación en 2011 de una tesina final de máster titulada «Proyecto y Topografía: Universidad Laboral de Málaga». En la actualidad, cursa el programa de Doctorado en Proyectos Arquitectónicos de la ETSA de Barcelona, dónde realiza su tesis doctoral con título «El Movimiento Moderno en las Universidades Laborales espańolas (1946-1978)», que ha tenido ocasión de presentar en diversas conferencias y congresos nacionales. Asimismo desde septiembre de 2012 colabora como investigador en el Grupo FORM del Departamento de Proyectos de la ETSA de Barcelona, en el proyecto del MICINN con título «Identificación, análisis y desarrollo de los componentes medioambientales del Movimiento Moderno en la arquitectura escolar en Brasil, Chile y México».

rcma.arq@gmail.com 